\title{
Article
}

\section{Intra-individual movement variability during skill transitions: A useful marker?}

Carson, H.J., Collins, D., and Richards, J.

Available at http://clok.uclan.ac.uk/11573/

Carson, H.J. ORCID: 0000-0002-3785-606X, Collins, D., ORCID: 0000-00027601-0454 and Richards, J. ORCID: 0000-0002-4004-3115 (2014) Intraindividual movement variability during skill transitions: A useful marker? European Journal of Sport Science, 14 (4). pp. 327-336. ISSN 1746-1391

It is advisable to refer to the publisher's version if you intend to cite from the work. http://dx.doi.org/10.1080/17461391.2013.814714

For more information about UCLan's research in this area go to http://www.uclan.ac.uk/researchgroups/ and search for < name of research Group>.

For information about Research generally at UCLan please go to http://www.uclan.ac.uk/research/

All outputs in CLoK are protected by Intellectual Property Rights law, including Copyright law. Copyright, IPR and Moral Rights for the works on this site are retained by the individual authors and/or other copyright owners. Terms and conditions for use of this material are defined in the policies page. 
ORIGINAL ARTICLE

3

4

5

6

Intra-Individual Movement Variability during Skill Transitions: A Useful Marker?

7

8 HOWIE J. CARSON ${ }^{1}$, DAVE COLLINS ${ }^{1}$, \& JIM RICHARDS ${ }^{2}$

$9 \quad{ }^{1}$ Institute for Coaching and Performance, University of Central Lancashire, Preston UK

${ }^{2}$ Allied Health Professions Research Unit, University of Central Lancashire, Preston, UK

23 Correspondence concerning this article should be addressed to Howie J. Carson, Institute for

24 Coaching and Performance, University of Central Lancashire, Greenbank Building room 161, 25 Preston, PR1 2HE. E-mail: HJCarson@uclan.ac.uk 


\section{Abstract}

Applied research suggests athletes and coaches need to be challenged in knowing when and how much a movement should be consciously attended to. This is exacerbated when the skill is in transition between two more stable states, such as when an already well learnt skill is being refined. Using existing theory and research, this paper highlights the potential application of movement variability as a tool to inform a coach's decision-making process when implementing a systematic approach to technical refinement. Of particular interest is the structure of co-variability between mechanical degrees-of-freedom (e.g., joints) within the movement system's entirety when undergoing a skill transition. Exemplar data from golf are presented, demonstrating the link between movement variability and mental effort as an important feature of automaticity, and thus intervention design throughout the different stages of refinement. Movement variability was shown to reduce when mental effort directed towards an individual aspect of the skill was high (target variable). The opposite pattern was apparent for variables unrelated to the technical refinement. Therefore, two related indicators, movement variability and mental effort, are offered as a basis through which the evaluation of automaticity during technical refinements may be made.

Keywords: Technical change, skill modification, skill refinement, conscious control, the Five-A Model, focus of attention 
 \\ Applied coaching: what the field needs}

For high-level performers of discrete skills, a crucial and unavoidable requirement is the ability to execute effective technique under high pressure conditions. As such, two important factors that must be considered when preparing a performer to compete, are the effectiveness of current technique, and its level of automaticity which, in turn, leads to resistance against the negative effects of pressure (Singer, 2002). While addressing the first of these factors represents a typical practice behaviour amongst high-level coaches, often by means of kinematic analyses to identify a particular weakness in technique (Bartlett, 2007) and evaluating performance outcome to understand its effect (Carson, Collins, \& MacNamara, in press), being able to assess movement automaticity presents a far greater challenge.

However, if available, such data would be useful for coaches when evaluating the progress of interventions in the build-up to high pressure situations. This is particularly pertinent, as we stress throughout this paper, in cases where an already existing and well-established technique is considered to be in need of refinement (cf. Carson \& Collins, 2011). In this regard, Carson and Collins define skill refinement as reflecting "the evolution of technique in a way that is new to the athlete" (p. 147), therefore indicating the necessity for transition from one original technique to an unfamiliar new version. Although this definition may initially sound rather drastic, it should be stressed that technical refinement is more often than not a subtle change or tweak to a specific aspect or component of technique. It is not, in contrast to skill acquisition, a process of establishing movement efficiency through coordination and control (cf. Newell, 1985); these variables having already been well learnt to good effect. In addition, from a theoretical perspective, such a "tool" could augment our ability to evaluate different learning and practice environments.

Reflecting this important task of refining technique, recent research has highlighted a significant gap within the literature addressing how a transition from one automated state to 
another may be achieved most effectively with long-term permanency and resistance to competitive pressure (Carson \& Collins, 2011). This is in stark contrast to either learning new skills, where automaticity is gradually acquired (Hays, Kornell, \& Bjork, 2010; Janelle, Champenoy, Coombes, \& Mousseau, 2003), or performing skills optimally through exploiting established automaticity (Beilock \& Gonso, 2008; Bell \& Hardy, 2009; Mesagno \& Mullane-Grant, 2010), where research is readily apparent. This gap has also been evidenced empirically within elite coaching practice, revealing unsystematic and inconsistent approaches employed by European Tour professional golfers and coaches when attempting to refine technique (Carson et al., in press). Crucially, Carson et al. discovered that interventions often lead to a lack of pressure resistance as well as regression back to the original technique, represented by constant fluctuations between automated and de-automated states, often over a period of several years. In practical terms, players and coaches appeared to be challenged in knowing when and how much the technique should be consciously attended to. This challenge was exacerbated when the skill was in transition between two more stable states, such as when an already well learnt and automated skill was being refined. Accordingly, golf presents a sound starting point from which to explore the promotion of effective skill refinement.

One potential line of enquiry in identifying the progress of refinement comes from the study of movement variability, accounting for "the normal variations that occur in motor performance across multiple repetitions of a task" (Stergiou \& Decker, 2011, p. 869). Previously, movement variability has been considered as the result of measurement "noise" (e.g., kinematic, kinetic). Notably, however, advances from a nonlinear dynamics perspective suggest that "it may be that the variance of movement dynamics is as revealing as, or more revealing than, the invariance in terms of unpacking the nature of the system organization" (Newell \& Slifkin, 1998, p. 157). Consequently, the need for evaluation and 
critical consideration of movement variability against the factor of automaticity is clear.

Indeed, and relevant to the current paper's focus on golf, recent reviews have focused on such study as an important route to an enhanced understanding of learning and performance (Glazier, 2011; Langdown, Bridge, \& Li, 2012).

Accordingly, in this paper we firstly examine areas of research that have explored the meaning behind movement and outcome variability as an indicator of skill level. Secondly, we draw upon the existing applied literature to propose how movement variability may be indicative of optimal or suboptimal performance states in high-level performers. This will be examined through a link with attentional focus, thus providing a reasoned prediction and measure for what could be expected when tracking the skill refinement process. Finally, exemplar data from golf are provided to show how, as a tool, this may be used to inform the process of refinement.

\section{Work in other areas: what is on offer?}

\section{Variability as a marker of skill learning}

From a process point of view, learning can be characterised as a progression towards outcome invariance associated with increasing performance-related attainment.

Concurrently, movement variability can also be employed as an indicator of learning or expertise as movement execution becomes more proficient (Gentile, 1972). However, unlike the recognised trend towards outcome invariance, the directional change (increased or decreased) in movement variability has formed the subject of much debate (e.g., Glazier, 2011; Newell \& Vaillancourt, 2001). For instance, Bradshaw et al. (2009) found higher skilled golfers to produce lower variability in key features of the golf swing (e.g., stance and timing) when compared to lower skilled golfers. In contrast, however, this trend of decreased movement variability associated with an increase in skill level, appears to be inconsistent across experimental findings and tasks. For example, Button, MacLeod, Sanders, and 
128 Coleman (2003) reported increased movement variability between the elbow and wrist joints during a basketball free throwing task when comparing experts' to novices' techniques prior to ball release. Clearly movement variability is a complex phenomenon when analysing the learning of skills, something that recent theory has attempted to explain.

\section{Resolving the problem of directional change: the uncontrolled manifold (UCM) hypothesis}

To better understand this complexity around the significance or meaning of directional change in movement variability, researchers have focused on one of Bernstein's (1967) most fundamental questions: that is, how does the motor system organise itself to solve a given task when a seemingly infinite number of combinations are available to it? Initially, Bernstein suggested that the central nervous system plans movement by constraining the many degrees-of-freedom (DoFs) into groups, or synergies, which are pertinent to achieving the task goal, whilst freezing or eliminating those that are not so essential. Glazier and Davids (2009) explain the formation of these synergies, as a reflection of lower skilled performers actively searching for stable (i.e., enduring and difficult to reform) and functional coordinative states. Therefore, from this perspective, motor planning requires eventually attending to a small(er) number of functional control variables, providing a simpler mechanism for movement organisation (Bernstein, 1967). However, in addition to the contradictory evidence from Button et al. (2003), some authors (e.g., Latash \& Anson, 2006) have argued against this notion, emphasising that freezing out DoFs requires perhaps enhanced control over certain joints, representing a far from trivial task. This point is a very important one and something that we shall return to in the next section.

Accordingly, if movement planning does not occur through the organisation of synergies and elimination of the remaining DoFs, what $i$ s actually happening? Recently, research has suggested that the answer can be found by considering two different, but equally important aspects of movement, stability and flexibility. A synergy is redefined as a 
structural unit (stability) that is also capable of error correction and adaptation (flexibility). In comparison to previous thought, the uncontrolled manifold (UCM) hypothesis (Scholz \& Schöner, 1999) seeks to identify motor synergies on the basis that no DoFs are ever frozen or eliminated but rather, that they are organised in such a way as to provide both stability and flexibility towards achieving specific task goals (Gelfand \& Latash, 1998). This is achieved by constraining (reducing the variability) the DoFs that are important to achieving the task goal, termed performance variables, into a structural unit, while at the same time releasing (thus increasing the variability) the DoFs that are not as important, termed elemental variables. As a result of this, the error-correction mechanism, or flexibility, to implement a synergy (movement pattern) within a variety of environmental contexts is now enabled. Accordingly, it is not the directional change of each individual DoF that is important but rather, the structure of co-variability between DoFs within the movement system's entirety (Langdown et al., 2012; Latash, Scholz, \& Schöner, 2002).

\section{Variability as a marker of transitions}

Similar to the nonlinear trends described when learning motor skills, recent evidence has demonstrated the potential for variability in performance results to be a useful indicator when experiencing a perturbation to an already well-established skill. Following the examination of successful olfactory and visual search refinement in dogs (i.e., the skill is already learnt, it simply requires a slight tweak), Helton (2011) concluded that, in order to facilitate long-term change in the dogs' ability to detect new stimuli, the existing (already well-established) detection strategy employed must be "overlaid" with an alternative one, directing attention towards the to-be-learnt stimuli. Following this, a shift towards consistent detection of the new stimuli manifested itself as a gradual fading out of the original strategy, representing a skill phase transition (a sudden and spontaneous shift in system components to form a new stable behaviour; Kelso, 1984). Data showed performance variability to steadily decrease 
and stabilise during the acquisition of the original behaviour. This was followed later by increases during the transitory stage and finally, by reduction back to original levels when restabilisation of the refinement had occurred. On the basis of these results, it seems that such patterns of change in performance (e.g., the number of fairways hit from tee shots in golf) could also be employed as a marker by coaches when tracking technical refinement in athletes.

\section{A summary of available perspectives}

The growth of interest in movement variability clearly reflects its potential to significantly contribute within research of applied coaching practice. However, its interpretation within the learning context appears to be, at present, very complex and strongly predicted by the interacting constraints described by Newell (i.e., organismic, task, and environmental; 1986), thus supporting a trend in favour of intra- as opposed to inter-individual analyses (e.g., Ball \& Best, 2012). Crucially however, in the case of either performance or elemental variables as described by the UCM, the amount of movement variability demonstrated by performers with a high level of automaticity should be relatively consistent (intra-individually) and, therefore, interpreted as entirely functional towards achieving a desired movement goal. Consequently, one may perhaps characterise the learning process more accurately as a move from dysfunctional to functional movement variability levels.

\section{Linking theory to practice: variability as a marker for refining already learnt skills}

Contrary to the volume of research on learning skills, there has been scarce consideration towards the expected intra-individual patterns of movement variability when undergoing transitory stages associated with a consciously initiated perturbation; for example when attempting a long-term permanent technical refinement once a high-level of skill and functional movement variability has already been established. However, several recent studies offer an insight into what can be expected. 


\section{Movement variability in applied settings}

Addressing the impact of movement variability from the applied literature, MacPherson, Collins, and Morriss (2008) suggest that when skilled performers exert a heightened level of conscious control, that is an internal focus (cf. McNevin, Shea, \& Wulf, 2003), to a single aspect of their technique, this results in decreased variability for that aspect, coupled with an increase in variability associated with other, less related movement constituents. This dysfunctional movement variability often leads to suboptimal levels of performance. To contextualise this finding against the UCM paradigm, the aspect subjected to increased conscious control decreases in variability because perhaps, temporarily at least, it is considered as more important than other aspects. Indeed, this would support the earlier contention of Latash and Anson (2006); dismissing the view that eliminating (reduced movement variability) a DoF represented an easier method of control. In fact, the results from MacPherson et al. (2008) would suggest the opposite!

It is worth addressing at this point somewhat of a contradiction within other attentional focus literature. In a recent review, Wulf (2013) suggested that an internal focus of attention served to constrain the motor system (reduce the variability), whereas an external focus releases the DoFs, therefore promoting functional movement variability that is much higher. While we support the notion that a specific internal focus would reduce the variability of that particular component, attention to the co-variability within the movement system as recommended by the UCM hypothesis appears to be lacking.

Accordingly, when applying these concepts relating to the optimum performance of movement skills to current models of refinement, we suggest that, once a movement has been learnt, movement variability "settles down" to a reasonably consistent, stable level. However, when the performer decides to work on a particular aspect of that movement by exerting increased conscious control, that particular part becomes more consistent (with even 
lower variability) whilst the variability of other non-associated parts increases. Once the change is fully re-automated and conscious control has been largely removed, variability levels return to a consistent and stable level across the different components of the skill (see Figure 1 for an idealised representation).

\section{Ensuring an adequate attentional focus}

When attempting to investigate the attentional focus-movement variability relationship, one important factor to consider is the performer's ability to apply a sufficient focus under both automated and de-automated conditions. Previous research into bimanual coordination suggests that movement of the upper-limbs are tightly coupled, with the brain deploying signals to the same muscle structures across both limbs as a default (Kelso, Southard, \& Goodman, 1979). Accordingly, symmetrical coordination of the limbs, known as in-phase, requires identical firing of muscle groups and reliably produces the most stable, automatic mode of coordination (Kelso, 1984; Zanone \& Kelso, 1992). In contrast, movements following an anti-phase pattern, alternated activation of the same muscle groups of each limb, are slightly less stable and require an increased attentional focus in order to stabilise (Temprado, Zanone, Monno, \& Laurent, 1999). The implications of these findings within the context of sports coaching is that changing, or disrupting, an already stabilised coordination pattern (consider this to represent an in-phase pattern) will be most effective if there is an attempt to de-couple the existing relationship between the left and right upperlimbs, should that be the desired modification. In other words, it is possible to apply a greater intensity of internal focus on one of the limbs in isolation rather than attending to both limbs simultaneously. As a result, this will likely serve to de-automate/destabilise the coordinative structure across the limbs via interference to the existing neural pathway. Therefore, this provides a theoretical and empirical basis on which to investigate the attentional focusmovement variability relationship. 
To exemplify how tracking trends in such a process may be utilised within the applied setting, we now provide a brief account of some pilot work in high-level golf examining the effect of attentional focus on movement co-variability. Based on the arguments presented above, we hypothesised that, when compared to the variability patterns observed in a wellknown and automated skill, increased (conscious) attention to a particular part of the skill would result in a decrease in variability. By contrast, and as another feature of this attention, the variability of non-crucial (i.e., not attended to) components would result in increased variability.

\section{What we might expect: exemplar cases of acute technical refinement in golf}

\section{Methods}

Participants. Three right handed male golfers between the ages of 25 and 30 years $(M=$ 26.7, $S D=2.9$ ) were recruited for this study. All were members of the Professional Golfers' Association (PGA) of Great Britain and Ireland. Preceding data collection, participants were required to read an information sheet and provide signed formal consent. Ethical approval was gained from the University's Ethics Committee prior to data collection.

Procedures. Prior to testing, participants were asked about their "natural" golf swing technique. It was established that two participants preferred to shape the golf ball in a left-toright direction (fade) and the remaining participant a right-to-left direction (draw) during play. All confirmed that to execute their natural technique would require a low level of conscious control; in other words, they could perform that particular type of shot with a high level of automaticity. After a warm-up phase of approximately five minutes, participants completed 10 full golf swings adopting their natural technique. To help promote automaticity, shots were executed with a commonly used golf club, a 7 iron, which was reported as easy to perform successfully, towards a distant target in a straight line. Prompts were provided after Trials 3, 6, and 9, to focus on hitting the target. Following these trials, 
participants discussed the changes in technique required to execute the non-preferred type of shot (i.e., fade when a draw was preferred, or vice versa); kinaesthetic cues were developed by each participant to help them detect the difference between the two techniques. Emphasis was placed on developing one key unilateral thought to focus on (a target variable) in order to bring about the desired change (cf. Kelso et al., 1979). As a result, all reported a focus towards the right arm movement during the backswing. Ten shots were then executed as per the previous condition, only this time participants were asked, and reminded after Trials 3,6 , and 9, to remain focused on their developed cue. Immediately following each of the two conditions, participants were asked to rate their overall level of mental effort (representative of conscious control) exerted during shot executions using the Rating Scale for Mental Effort (Zijlstra, 1993). The scale ranged from 0 (not at all effortful), to 75 (moderately effortful), and 150 (very effortful). For the second condition, this reflected the level of awareness directed towards the kinaesthetic cue aimed at changing the target variable. All kinematic data were collected using an inertial-sensor motion capture suit (MVN Biomech Suit, Xsens ${ }^{\circledR}$ Technologies B.V., Enschede, The Netherlands) at a sampling rate of $120 \mathrm{~Hz}$.

Data processing and analysis. Raw data from the MVN Studio Software (Xsens ${ }^{\circledR}$ Technologies B.V, Enschede, the Netherlands) were exported into c3d file format and analysed using six degrees of freedom modelling with Visual3 $\mathrm{D}^{\mathrm{TM}}$ v4.89.0 software $(\mathrm{C}$ Motion ${ }^{\circledR}$ Inc, Germantown, MD, USA). Two swing events were identified to define the backswing, with the time between each event normalised to 101 points. The first event (onset) was defined as the frame when the left hand's centre of gravity linear speed crossed a threshold value of $0.2 \mathrm{~m} / \mathrm{s}$ in the local medial/lateral axis relative to pelvis. The second event (top of swing) was defined as the frame when the distal end position of the right hand reached its maximum value in the global vertical axis. All data were exported to Microsoft Excel ${ }^{\circledR}$ 2010 for graphical analysis of variables related to the right and left upper-limbs. 

that were related (i.e., the right upper-limb; target variable) and unrelated (i.e., the left upperlimb; a non-target variable) to the technical refinement.

Results

Mental effort ratings increased for all participants between the initial target focus (low mental effort) and second unilateral internal focus (high mental effort) conditions; results are presented in Figure 2. Movement variability showed a decrease in the right elbow for all participants during the high mental effort condition, where there was an explicit focus on the kinaesthesia of the right arm (see Figure 3 left column). In association with directing attention to this unilateral movement constituent, and as predicted, movement variability increased for left upper-limb joints (see Figure 3 right column). Changes in kinematic joint angles are presented in Figure 4, evidencing that changes intended in the second condition were actually achieved. One distinct feature of these graphs is the inter-individual nature of change for both variability and kinematic measures. As such, statistical treatment of data was seen as inappropriate.

\section{Discussion}

These exemplar cases aimed to examine the implementation of intra-individual movement variability when addressing technical refinement against a factor of conscious control within a single session. In doing so, kinematic analyses provide insightful data to support the suggested patterns of movement variability during this transitory process, especially when considered against the theoretical suggestions of the UCM hypothesis (cf. Scholz \& Schöner, 1999). What is important to highlight at this early stage of experimentation, is our intention not to provide a test of the UCM hypothesis, but rather to use its insights into movement planning and organisation to help interpret our data and guide applied practice. In addition, the data support previous findings that show a decrease in movement variability when an 
internal focus is applied (cf. Wulf, 2013). Furthermore, they reveal that the structure of variability across related and unrelated variables is highly complex, supporting the need for intra-individual analyses, but which can indeed inform about the nature of the motor system's organisation (Newell \& Slifkin, 1998).

Data support the underlying importance of tracking kinematic factors to determine a stable level of execution or level of automaticity for complex movements (MacPherson et al., 2008), and could also be viewed as support towards the progression of events across the attractor landscape over multiple time scales, as described by Newell, Liu, and Mayer-Kress (2001). This is a crucial point within coaching practice since describing the motor system at a behavioural level (i.e., analysis of technique) will not provide any indication towards the level of automaticity or stability within the system evolving over the course of such a dynamic transitory process. Hence, as mentioned within the introduction, being able to assess both factors of execution remains essential when assessing the refinement of skills, since one would demonstrate the actual execution of correct technique (location along the attractor landscape) before it was able to be performed with high levels of automaticity (depth of the attractor well). Indeed, analysis of performance (cf. Helton, 2011) may also prove to reveal a longer-term timescale for refinement at an outcome level. In short, it is unrealistic to expect long-term pressure resistant technical change to result from a single session of practice.

From a practical point of view, by measuring movement variability against mental effort, two process markers are provided, enabling greater triangulation (along with conventional outcome data) of information which, in turn, can be used to better inform coaching decisions and, from a research perspective, track change under different practice conditions. 
One limitation of the data presented is the lack of detailed consideration towards the co-variation between several joints across a coordinative structure (e.g., multiple joints of the same limb), nor between axes of rotation relating to each of the target and non-target variables. An analysis of co-variability across proximal-to-distal joint couplings may prove additionally insightful, especially when adopting a focus that is either more proximal (e.g., the left elbow) or distal (e.g., the left wrist) to the movement's centre. Indeed, this is something that future research should investigate. However, in the case of highly asymmetric movements such as the golf swing, assessing the co-variability between joints across both limbs (i.e., flexion-extension, internal-external rotation, and add-abduction of the left and right elbows for instance) may not prove as useful since it may not be possible, or even the desired technical refinement, to individually constrain the axes of rotation about a joint as a direct function of attentional focus. Nor will the corresponding axes of rotation about opposing joints (e.g., left and right elbows) necessarily be coupled when performing the golf swing. However, this may be of interest when examining in-phase movements typical of laboratory experiments (e.g., Zanone \& Kelso, 1992). What these data do support is the potential use of movement variability directed towards the general area, but that is locked into the performer's focus of attention. Therefore, from a coaching perspective, providing each variable, target and non-target, remain on the course of variability pattern as depicted in Figure 1; both would present appropriate markers for tracking the skill refinement process. In viewing the significant and robust contribution that may be gained from employing an analysis using the UCM method, this study is limited by not doing so; however, is something that experimenters may wish to consider. Indeed, our own future work will aim to include some elements of this testing in representative performance environments.

Principally, there were several reasons to explain its exclusion from the present study. We were not able to conclusively identify success in achieving a predetermined position of the 
target variable. Rather, this was related to the performer's ability to reproduce the selfgenerated kinaesthesia. When conducting an analysis using the Uncontrolled Manifold method, Scholz, Schöner, and Latash (2000) state that mixing successful and unsuccessful trials would not makes sense since they correspond to different manifolds. With the possibility for this mixture within our data, we considered such an analysis as potentially flawed. The authors also later explain that to perform such an analysis would require sufficiently more trials than we have collected, namely 20 (Latash, Levin, Scholz, \& Schöner, 2010). Accordingly, and in contrast to the methods reported in this study, greater efforts would need to be focused on predefining a task variable (e.g., golf club position or exact positioning of a target variable) to be able to compare between successful and unsuccessful trials. This would therefore facilitate an analysis of different hypotheses to determine which variables were considered to provide stability or flexibility to the technique. To obtain a detailed examination of this method in a comparable scenario, pistol shooting, we encourage those interested to read the paper by Scholz et al. (2000) who compared the impact of different variables on shooting success. What we hope to have achieved in this paper is to establish a formal link between the structure of a movement synergy and the intensity and direction of a performer's attentional focus (conscious control/automaticity).

\section{Conclusion}

By adopting the theoretical standpoint offered by the UCM hypothesis, it is clear that attention in measurement must be paid towards the structure of movement variability or, in other words, the co-variability across different components of a skill when addressing technical refinement. In using this approach, an examination into the effects of associated attentional foci on movement kinematics during the process of refinement has been made. Therefore, when movement variability and mental effort are measured in tandem, a coach, most probably through assistance from applied sport science support (cf. Carson et al., in 
press), may be better informed about a performer's level of automaticity and readiness to compete. What is now required to verify these contentions and initial findings is to implement and assess the practical use of movement variability over an extended time period within an applied coaching framework, and across a variety of changes and performers when undergoing a planned technical refinement. In doing so, this may provide more robust

407 evidence towards the theoretical meaning and operational use of movement variability. In 408 sum, this paper highlights the need for an understanding of movement variability as an index 409 of attentional focus when implementing technical refinements in applied coaching practice. 
410

411

412

413

414

415

416

417

418

419

420

421

422

423

424

425

426

427

428

429

430

431

432

433

434

\section{References}

Ball, K., \& Best, R. (2012). Centre of pressure patterns in the golf swing: Individual-based analysis. Sports Biomechanics, 11, 175-189.

Bartlett, R. (2007). Introduction to sports biomechanics: Analysing human movement patterns (2nd ed.). Abingdon: Routledge.

Beilock, S. L., \& Gonso, S. (2008). Putting in the mind versus putting on the green: Expertise, performance time, and the linking of imagery and action. The Quarterly Journal of Experimental Psychology, 61, 920 - 932.

Bell, J. J., \& Hardy, J. (2009). Effects of attentional focus on skilled performance in golf. Journal of Applied Sport Psychology, 21, 163-177.

Bernstein, N. A. (1967). The coordination and regulation of movements. Oxford: Pergamon Press.

Bradshaw, E. J., Keogh, J. W. L., Hume, P. A., Maulder, P. S., Nortje, J., \& Marnewick, M. (2009). The effect of biological movement variability on the performance of the golf swing in high- and low-handicapped golfers. Research Quarterly for Exercise and Sport, 80, 185-196.

Button, C., MacLeod, M., Sanders, R., \& Coleman, S. (2003). Examining movement variability in the basketball free-throw action at different skill levels. Research Quarterly for Exercise and Sport, 74, 257-269.

Carson, H. J., \& Collins, D. (2011). Refining and regaining skills in fixation/diversification stage performers: The Five-A Model. International Review of Sport and Exercise Psychology, 4, 146-167.

Carson, H. J., Collins, D., \& MacNamara, Á. (in press). Systems for technical refinement in experienced performers: The case from expert-level golf. International Journal of Golf Science. 
Gelfand, I. M., \& Latash, M. L. (1998). On the problem of adequate language in motor control. Motor control, 2, 306-313.

Gentile, A. M. (1972). A working model of skill acquisition with application to teaching. Quest, 17, 3-23.

Glazier, P. (2011). Movement variability in the golf swing: Theoretical, methodological, and practical issues. Research Quarterly for Exercise and Sport, 82, 157-161.

Glazier, P. S., \& Davids, K. (2009). On analysing and interpreting variability in motor output. Journal of Science and Medicine in Sport, 12, e2-e3.

Hays, M. J., Kornell, N., \& Bjork, R. A. (2010). Costs and benefits of feedback during learning. Psychonomic Bulletin and Review, 17, 797-801.

Helton, W. S. (2011). Animal expertise: Evidence of phase transitions by utilizing running estimates of performance variability. Ecological Psychology, 23, 59-75.

Janelle, C. M., Champenoy, J. D., Coombes, S. A., \& Mousseau, M. B. (2003). Mechanisms of attentional cueing during observational learning to facilitate motor skill acquisition. Journal of Sports Sciences, 21, 825-838.

Kelso, J. A. S. (1984). Phase transitions and critical behavior in human bimanual coordination. American Journal of Physiology, 246, R1000-R1004.

Kelso, J. A. S., Southard, D. L., \& Goodman, D. (1979). On the coordination of two-handed movements. Journal of Experimental Psychology: Human Perception and Performance, 5, 229-238.

Langdown, B. L., Bridge, M., \& Li, F.-X. (2012). Movement variability in the golf swing. Sports Biomechanics, 11, 273-287.

Latash, M. L., \& Anson, J. G. (2006). Synergies in health and disease: relations to adaptive changes in motor coordination. Physical Therapy, 86, 1151-1160. 
Latash, M. L., Levin, M. F., Scholz, J. P., \& Schöner, G. (2010). Motor control theories and their applications. Medicina (Kaunas), 46, 382-392.

Latash, M. L., Scholz, J. P., \& Schöner, G. (2002). Motor control strategies revealed in the structure of motor variability. Exercise and Sport Sciences Reviews, 30, $26-31$.

MacPherson, A. C., Collins, D., \& Morriss, C. (2008). Is what you think what you get? Optimizing mental focus for technical performance. The Sport Psychologist, 22, 288303.

McNevin, N. H., Shea, C. H., \& Wulf, G. (2003). Increasing the distance of an external focus of attention enhances learning. Psychological Research, 67, 22-29.

Mesagno, C., \& Mullane-Grant, T. (2010). A comparison of different pre-performance routines as possible choking interventions. Journal of Applied Sport Psychology, 22, $343-360$.

Newell, K. M. (1985). Coordination, control and skill. In D. Goodman, R. B. Wilberg \& I. M. Franks (Eds.), Differing perspectives in motor learning, memory, and control (pp. 295 - 317). Amsterdam: Elsevier Science.

Newell, K. M. (1986). Constraints to the development of coordination. In M. G. Wade \& H. T. A. Whiting (Eds.), Motor development in children: Aspects of coordination and control (pp. 341-360). Dordrecht, The Netherlands: Martinus Nijhoff.

Newell, K. M., Liu, Y.-T., \& Mayer-Kress, G. (2001). Time scales in motor learning and development. Psychological Review, 108, 57-82.

Newell, K. M., \& Slifkin, A. B. (1998). The nature of movement variability. In J. P. Piek (Ed.), Motor behavior and human skill: A multidisciplinary approach (pp. 143-160). Champaign, IL: Human Kinetics.

Newell, K. M., \& Vaillancourt, D. E. (2001). Dimensional change in motor learning. Human Movement Science, 20, 695-715. 

606

Scholz, J. P., \& Schöner, G. (1999). The uncontrolled manifold concept: Identifying control variables for a functional task. Experimental Brain Research, 126, 289-306.

Scholz, J. P., Schöner, G., \& Latash, M. L. (2000). Identifying the control structure of multijoint coordination during pistol shooting. Experimental Brain Research, 135, $382-404$.

Singer, R. N. (2002). Preperformance state, routines, and automaticity: what does it take to realize expertise in self-paced events? Journal of Sport and Exercise Psychology, 24, $359-375$.

Stergiou, N., \& Decker, L. M. (2011). Human movement variability, nonlinear dynamics, and pathology: Is there a connection? Human Movement Science, 30, 869-888.

Temprado, J. J., Zanone, P. G., Monno, A., \& Laurent, M. (1999). Attentional load associated with performing and stabilizing preferred bimanual patterns. Journal of Experimental Psychology: Human Perception and Performance, 25, 1579-1594.

Wulf, G. (2013). Attentional focus and motor learning: A review of 15 years. International Review of Sport and Exercise Psychology, 6, 77-104.

Zanone, P. G., \& Kelso, J. A. S. (1992). Evolution of behavioural attractors with learning: Nonequilibrium phase transitions. Journal of Experimental Psychology: Human Perception and Performance, 18, 403-421.

Zijlstra, F. R. H. (1993). Efficiency in work behaviour: A design approach for modern tools. Delft: Delft University Press.

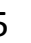




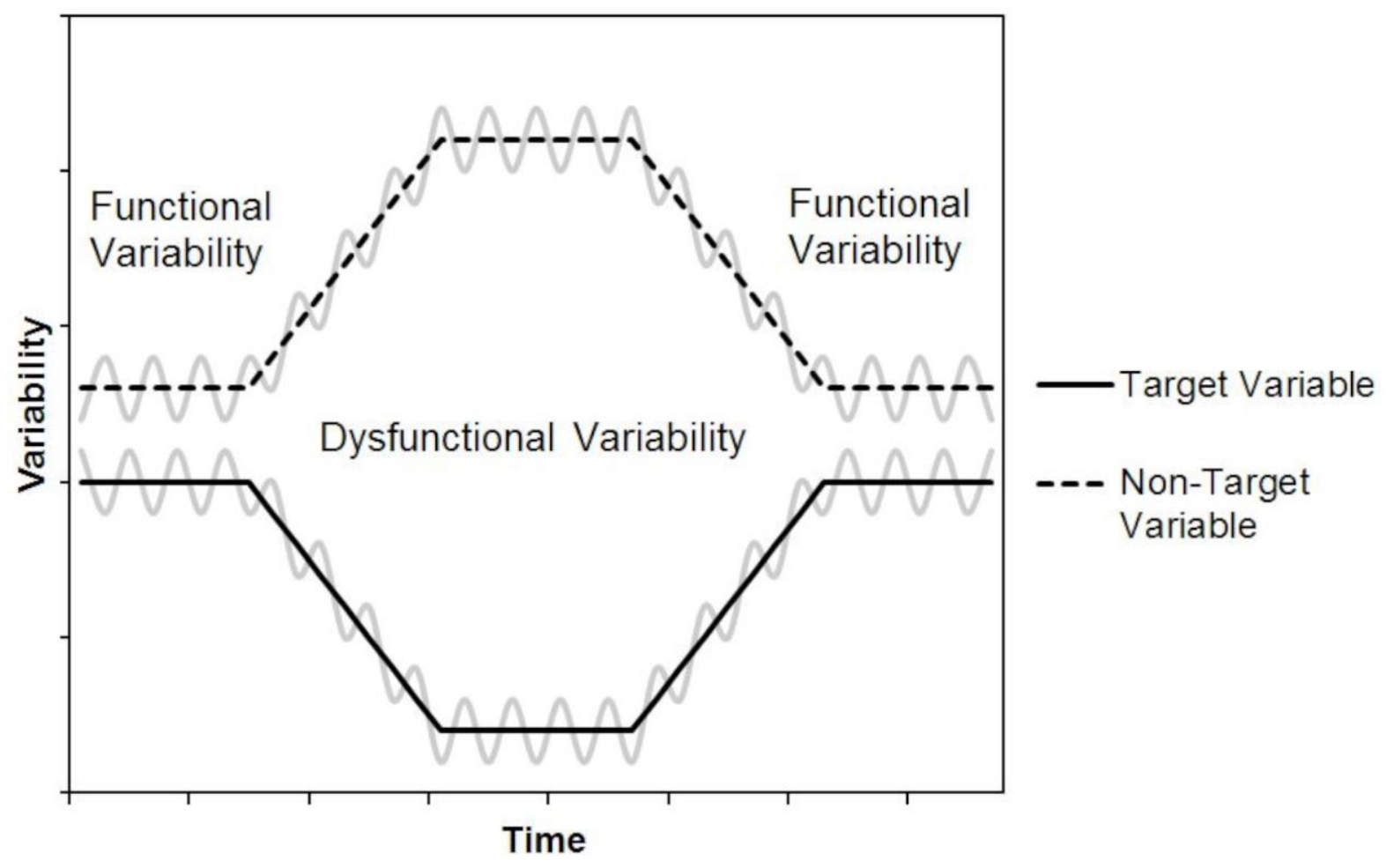

$510 \quad$ Figure 1.

511

512

513

514

515

516

517

518

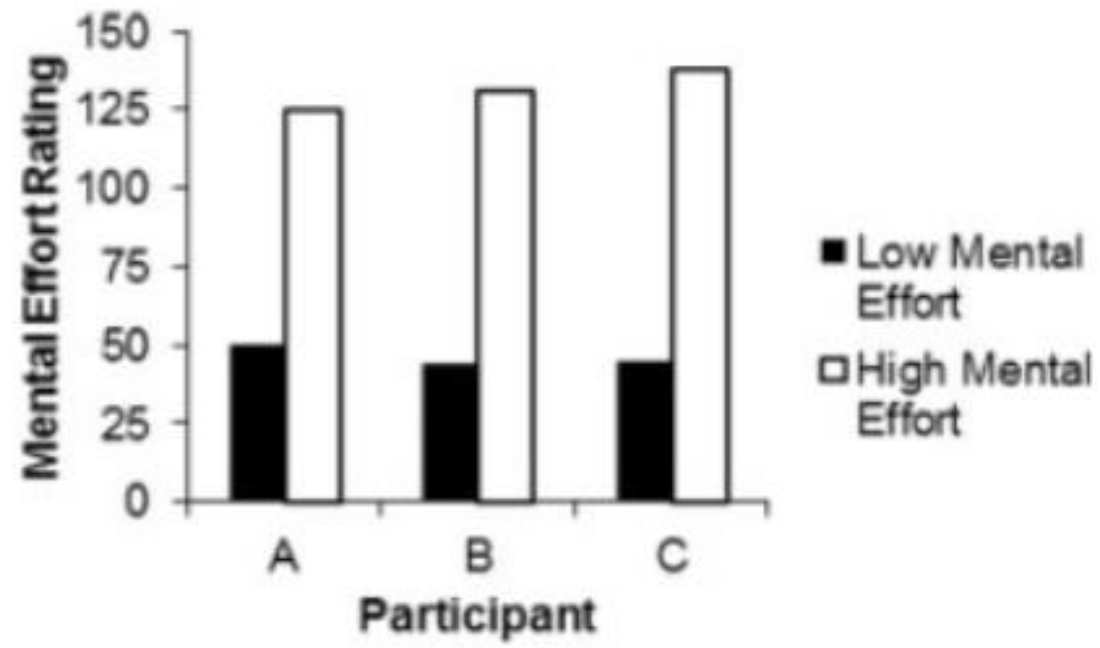

519

520 Figure 2. 


\section{Target Variable}

A

Right Elbow Flexion-Extension

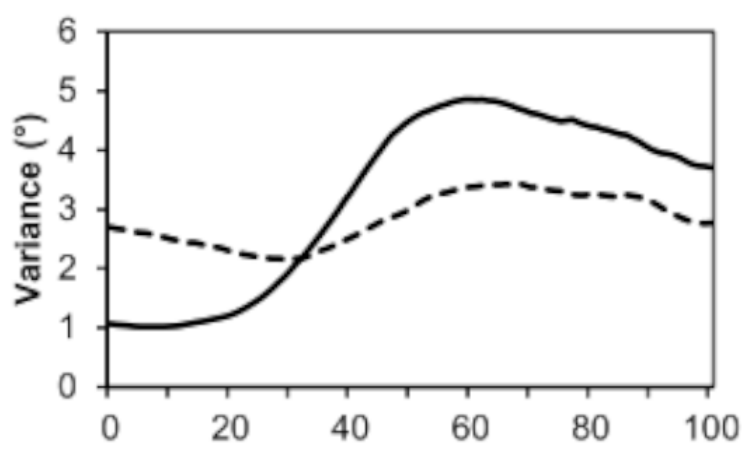

B

Right Elbow Add-Abduction

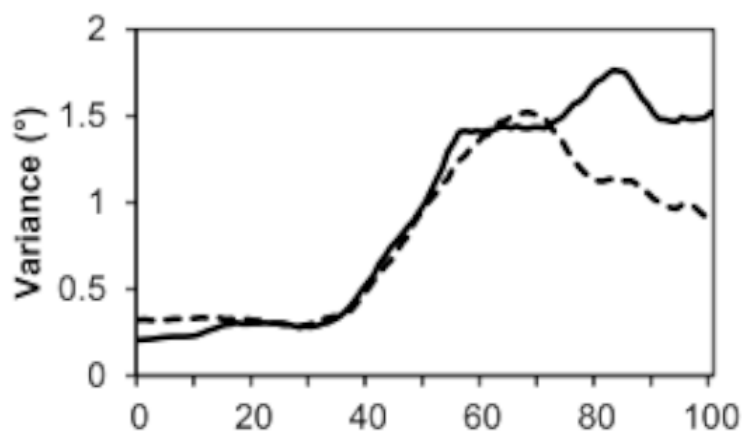

C

Right Elbow Add-Abduction

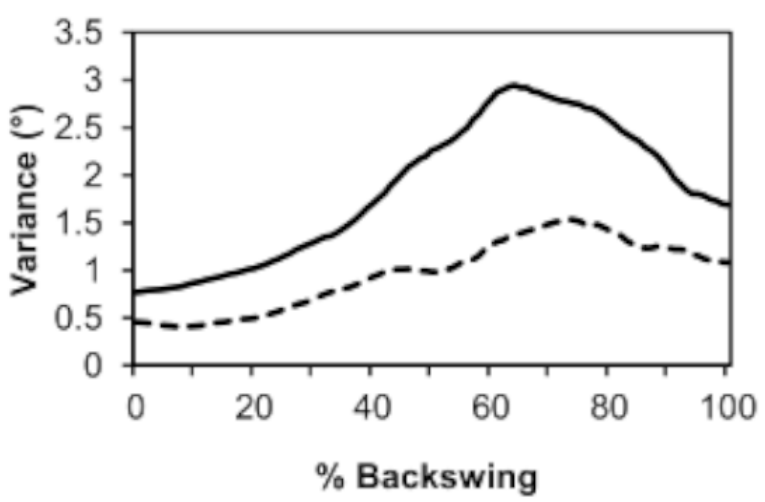

Non-Target Variable

Left Elbow Add-Abduction

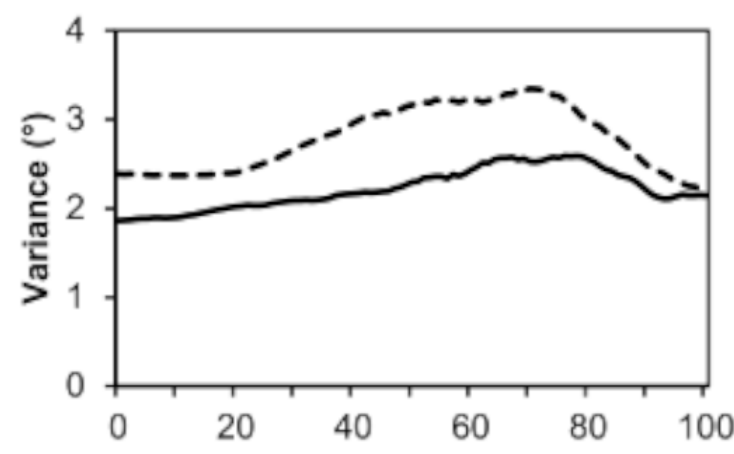

Left Elbow Int-External Rotation

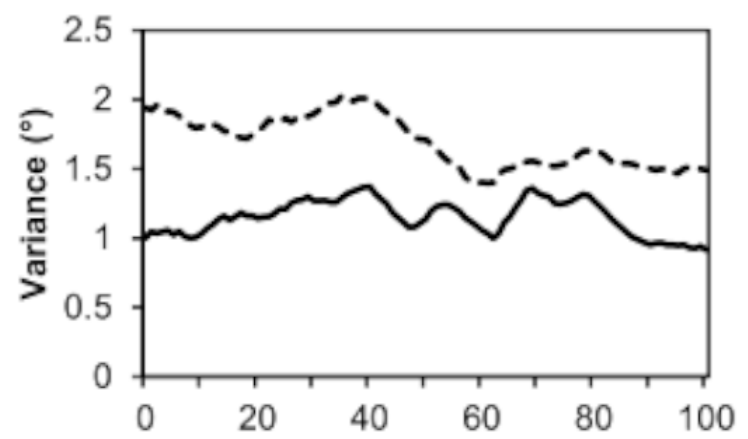

Left Wrist Int-External Rotation

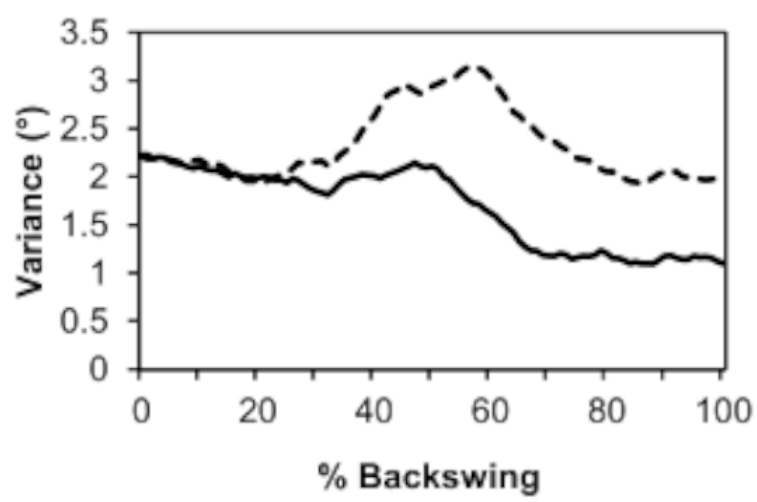

522 Figure 3. 


\section{Target Variable}

A

Right Elbow Flexion-Extension

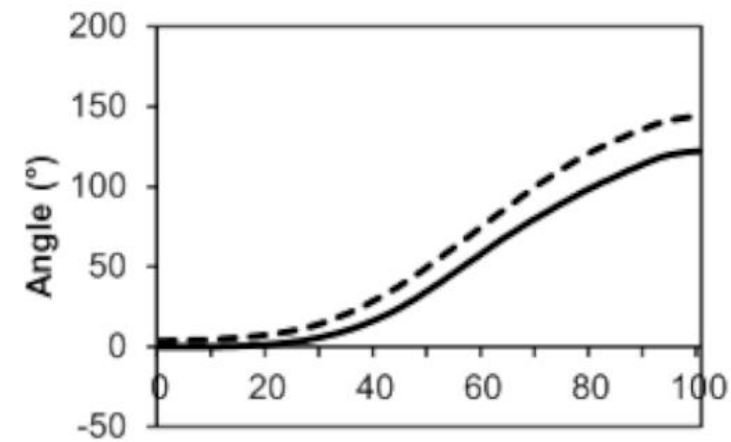

B

Right Elbow Add-Abduction

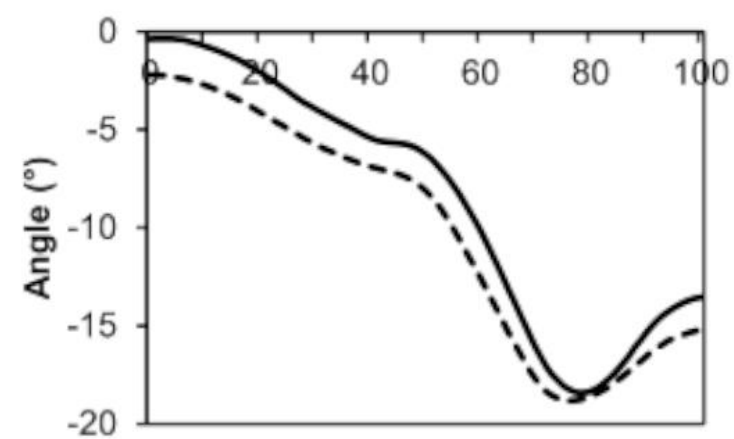

C

Right Elbow Add-Abduction

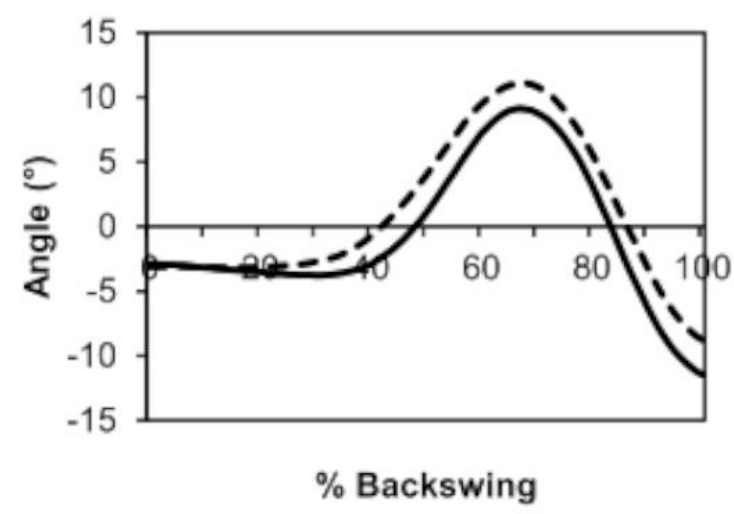

Non-Target Variable

Left Elbow Add-Abduction

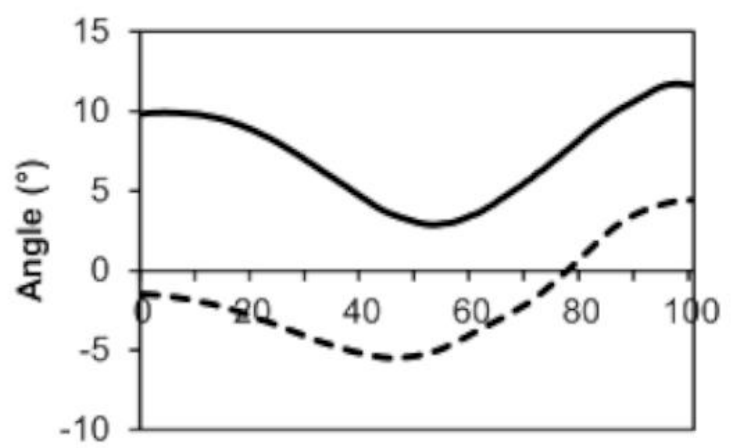

Left Elbow Int-External Rotation

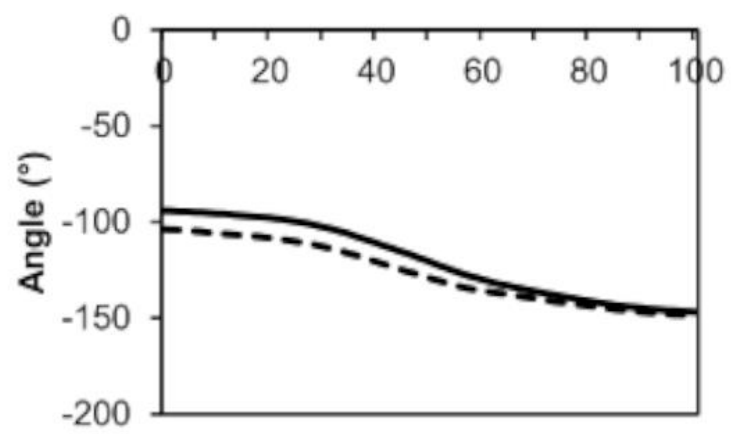

Left Wrist Int-External Rotation

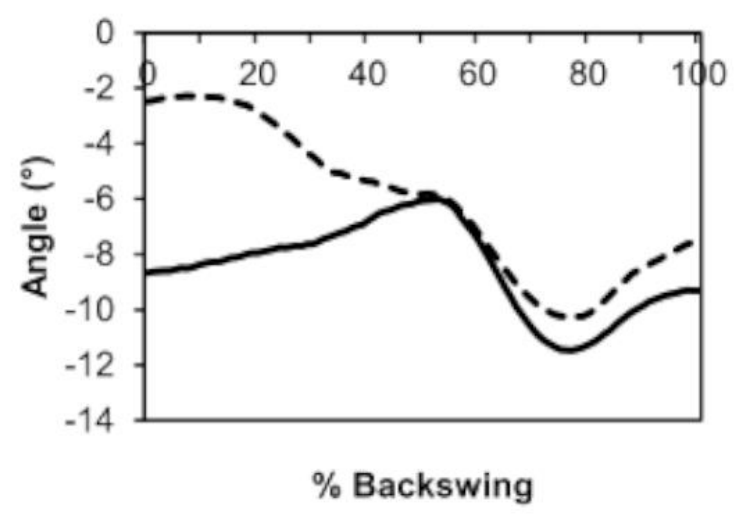

Figure 4. 


\section{$528 \quad$ Figure captions}

529

530

531

532

533

534

535

536

537

538

539

540

541

542

543

544

545

546

547

- $\quad$ Figure 1. An idealised representation of co-variability through the refinement process, depicting initially stable and consistent levels of variability for two components of a movement (functional variability). As one of those components is consciously attended to (target variable), movement variability decreases for that component associated with an increase in variability for the non-targeted component (dysfunctional variability). Due to the levels of dysfunctional movement variability being inherently unknown within each individual, completion of this phase is characterised by a levelling out in variability, signifying maximum de-automation. Gradual automation of the new technique is shown to occur through a stable return to largely subconscious thought and functional variability of both movement components. Reflecting the inherent nonlinear nature of this process, the faint lines depict a more representative data set with the straight lines representing trends.

- $\quad$ Figure 2. Mental effort scores when performing under initially low and then high levels of mental effort directed towards a target variable.

- $\quad$ Figure 3. Movement co-variance for kinematics subjected to an increase in conscious control relating to the right limb (target variable) and less associated variables relating to the left limb (non-target variable), measured from the swing onset to the top of the backswing.

- $\quad$ Figure 4. Mean positional data for the target and non-target variables measured from the swing onset to the top of the backswing. 